\title{
Reaction of substituted 6-oxocyclohexane- 1,3-dicarboxamides with hydroxylamine
}

\author{
(C) Natalia V. Nosova, Daria D. Lezhnina, Daria D. Rubtsova, and Vladimir L. Gein** \\ Department of General and Organic Chemistry. Perm State Pharmaceutical Academy. \\ Polevaya, St., 2.Perm, 614090. Russia.Phone: +7 (342) 236-90-50.Email: geinvl48@mail.ru
}

\begin{abstract}
*Supervising author; ${ }^{+}$Corresponding author Keywords: 6-oxocyclohexane-1,3-dicarboxamides, hydroxylamine, oximes, 2-aryl-4-hydroxy-6(hydroxyimino)-4-methylcyclohexane-1,3-dicarboxamides.
\end{abstract}

\begin{abstract}
Functionalized cyclohexanones are multifunctional highly reactive available substrates. They are convenient for the synthesis of new compounds, including practically significant ones. The presence of a 1,3dicarbonyl fragment in their composition makes it possible to obtain various heterocyclic systems in reactions with binucleophilic reagents: indazoles, benzisoxazoles, and diazepines. The interaction of cyclohexanones functionalized with acetyl or alkoxycarbonyl substituents with hydroxylamine was previously studied. It was shown that, depending on the nature of the substituents, the reaction can proceed with the formation of the corresponding oximes, or give products of heterocyclization - benzisoxazoles. The interaction of cyclohexanones containing amide groups in an alicycle with hydroxylamine has not been studied. We studied the reaction of unsubstituted 2-aryl-4-hydroxy-4-methyl-6-oxocyclohexane-1,3-dicarboxamides with hydroxylamine hydrochloride in the presence of an equivalent amount of alkali. When boiling in ethanol in the absence of a catalyst, new 2-aryl-4-hydroxy-6-(hydroxyimino)-4-methylcyclohexane-1,3-dicarboxamides were obtained. Cyclization with the formation of benzisoxazoles does not occur in this case due to a lower positive charge on the carbon atom of the amide group compared to the carbon atom of the acetyl or alkoxycarbonyl groups. The structure of the synthesized compounds was established based on the data of IR, ${ }^{1} \mathrm{H}$ NMR and ${ }^{13} \mathrm{C}$ NMR spectroscopy. The IR spectra of the obtained oximes contain bands of valence vibrations of the $\mathrm{OH}, \mathrm{NH}$, and $\mathrm{CON}$ groups. The ${ }^{1} \mathrm{H}$ NMR spectra contain four NH-proton singlets of two amide groups, the proton singlet $=\mathrm{N}-\mathrm{OH}$ group. The ${ }^{13} \mathrm{C}$ NMR spectra of solutions of compounds contain signals of two carbon atoms of amide groups and a carbon atom of the $\mathrm{C}=\mathrm{N}-\mathrm{OH}$ group, which fully confirm the assumed structure.
\end{abstract}

\section{References}

[1] A.K. Murthy, K.S.R.K.M. Rao, Rao N.V. Subba. Synthesis and physiological activity of hydroxyaryl isoxazoles. J. Indian Chem. Soc. 1972. Vol.42. No.10. P.1025-1028.

[2] C.J. Rao, A.K. Murthy. Fused heterocycles. Part I. Synthesis of 7,8-dihydro-6H-3-methyl-5,7diarylisoxazolo[4,5-b]azepines. Indian J. Chem. 1978. B16. No.7. P.636-637.

[3] N.O. Smirnova, O.P. Plotnikov, N.A. Vinogradova, V.V. Sorokin, A.P. Kriven 'ko. Synthesis and biological activity of substituted 7-aza-8-aza(oxa)dicyclo[4,3,0,]nonadienes-6,9. Pharm. Chem. J. 1995. Vol.1. P.44-46. (russian)

[4] V.L. Gein, N.V. Nosova, A.V. Vagapov, N.V. Dozmorova, A.N. Yankin. Reactions of 2-acetyl-5hydroxy-5-methyl-3-phenylcyclohexanone and alkyl-4-hydroxy-4-methyl-2-oxo-6phenylcyclohexanecarboxylates with nucleophilic reagents. Russ. J. of Gen. Chem. 2019. Vol.89. No.7. P.987-994. (russian)

[5] V.L. Gein, N.V. Nosova, K.D. Potemkin, Z.G. Alyev, A.P. Kriven'ko. Synthesis, structure and reactions of diisopropyl-2-R-6-hydroxy-6-methyl-4-oxocyclohexane-1,3-dicarboxylates with nucleophilic reagents. Russ. J. of Org. Chem. 2005. Vol.41. No.7. P.1039-1045. (russian) 